\title{
Correction to: Application of Mathematical Modelling as a Tool to Analyze the EEG Signals in Rat Model of Focal Cerebral Ischemia
}

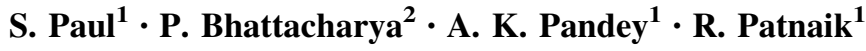

Published online: 30 April 2020

(C) The Institution of Engineers (India) 2020

Publisher's Note Springer Nature remains neutral with regard to jurisdictional claims in published maps and institutional affiliations.

Correction to: J. Inst. Eng. India Ser. B

(January-March 2014) 95(1):23-27

https://doi.org/10.1007/s40031-014-0072-5

In the original publication of the article, figure 1 was published incorrectly. The corrected figure is given below (Fig. 1):

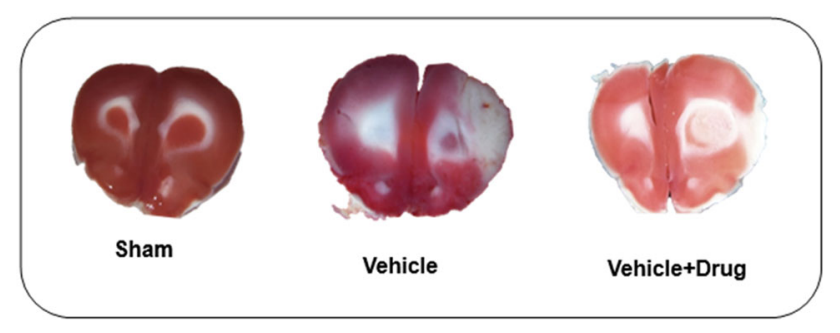

Fig. 1 TTC stained $2 \mathrm{~mm}$ coronal rat brain sections of Sham, Vehicle and Piroxicam (10 mg/kg b.w) treated with $1 / 24 \mathrm{~h} \mathrm{I} / \mathrm{R}$ injury

The original article can be found online at https://doi.org/10.1007/s40031-014-0072-5.

S. Paul

sudip.paul.bhu@gmail.com

1 School of Biomedical Engineering, Indian Institute of Technology, Banaras Hindu University, Varanasi, Uttar Pradesh 221005, India

2 Department of Neurology, Leonard M. Miller School of Medicine, University of Miami, Miami, FL 33136, USA 